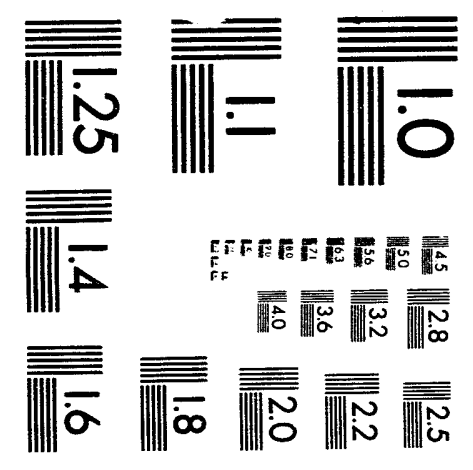



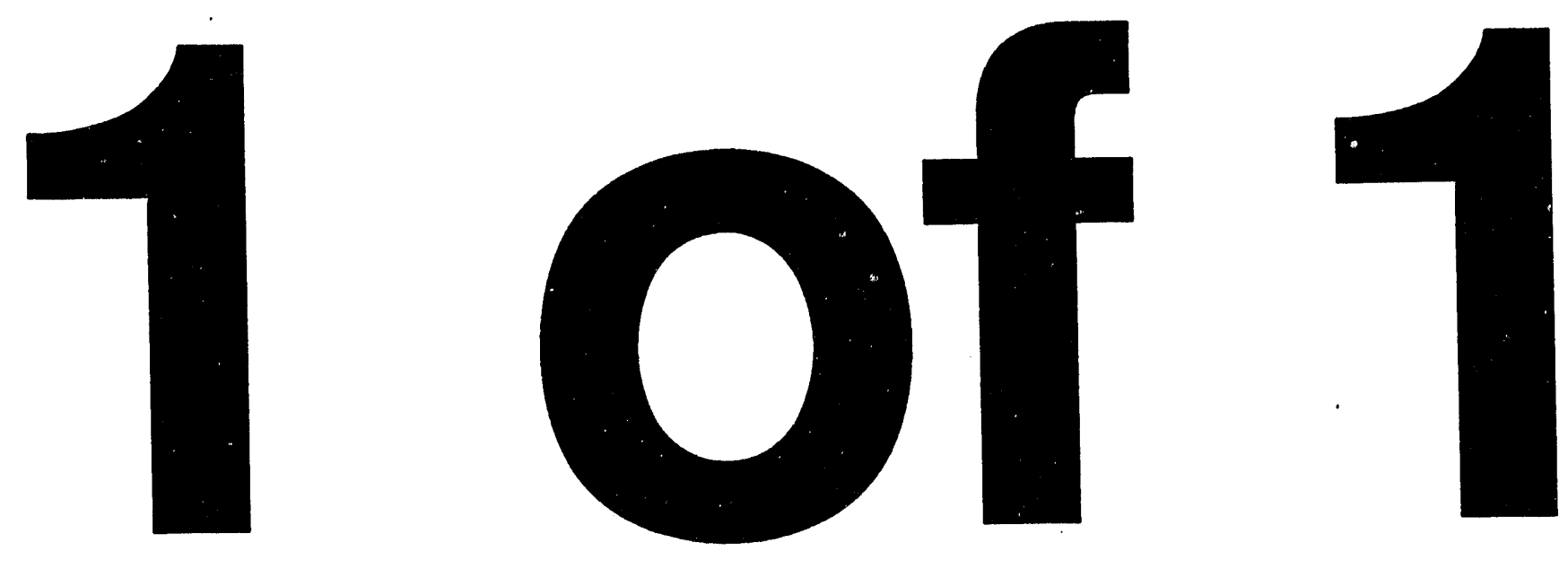
TITLE: Munitions Classification Using an Acoustic Resonance Spectroscopic Technique

AUTHOR(S): Randy S. Roberts, Jerome T. Chen, Octavio A. Vela, Pau1 S. Lewis

SUBMITTED TO: 1993 Asilomar Conference

\section{DISCLAIMER}

This report was prepared as an account of work sponsored by an agency of the United States Government. Neither the United States Government nor any agency thereof, nor any of their employees, makes any warranty, express or implied, or assumes any legal liability or responsibility for the accuracy, completeness, or usefulness of any information, apparatus, product, or process disclosed, or represents that its use would not infringe privately owned rights. Reference herein to any specific commercial product, process, or service by trade name, trademark, manufacturer, or otherwise does not necessarily constitute or imply its endorsement, recommendation, or favoring by the United States Government or any agency thereof. The views and opinions of authors expressed herein do not necessarily state or reflect those of the United States Government or any agency thereof. 


\title{
Munitions Classification using an Acoustic Resonance Spectroscopic Technique
}

\author{
Randy S. Roberts Jerome T. Chen Octavio A. Vela Paul S. Lewis \\ Group ESA-6, Mail Stop J580 \\ Los Alamos National Laboratory \\ Los Alamos, NM 87545
}

\begin{abstract}
A nondestructive evaluation technique for classifying artillery munitions is presented. This technique, based on acoustic resonance measurements, has been successfully demonstrated on a wide variety of high explosive and chemical munitions. The technique consists of building templates of spectral features from sets of known munitions. Spectral features of unknown $m u$. nitions are compared with a library of templates, and the degree of match between the features and the templates is used to classify the munition. This paper describes the technique, including the feature extraction, clustering and classification algorithms.
\end{abstract}

\section{Introduction}

In support of the Bilateral Chemical Weapons Agreement between the United States and Russia, Los Alamos National Laboratory has developed a nondestructive evaluation (NDE) technique that discriminates between different types of artillery munitions. This NDE classification technique allows on-site inspectors to rapidly classify the munitions as chemical or high explosive, and furthermore discriminates between various subclasses of these types of munitions. It provides an alternative to time consuming or hazardous techniques such as drill-and-sample. The technique, based on Acoustic Resonance Spectroscopy (ARS), has been successfully field tested on a wide variety of munitions [1].

Acoustic Resonance Spectroscopy is the study of the mechanical resonance structure of objects. Objects that are composed of elastic materials have natural modes of vibration. These modes are standing

- This work was supported by the Defense Nuclear Agency waves in the object and can be excited by applicavion of a vibration at the appropriate frequencies. These frequencies, called resonant frequencies, are determined by the geometry of the object as well as its material composition. The munitions specified in the bilateral agreement have unique sets of resonant peaks in their acoustic spectrum. These peaks, as previously noted, are related to the mechanical structure of the munition and can be used to uniquely identify a particular class of munition. For example, munitions with thinner walls tend to produce acoustic resonances at higher frequencies than those with thicker wails. Munitions filled with solids tend to produce broader acoustic resonances than those filled with a liquid. Likewise, munitions filled with viscous liquids tend to produce wider resonant peaks than those with lighter liquids. It is this type of difference in ar oustic spectra that provide the foundation of the ARS classification technique.

The ARS classification tecliniqlie constructs templates of spectral features from 5 nown munition classes and compares the teraplater with the spectral features of an unknown wait; a. To construct a template, spectral data fror a a inumino $n$ the training

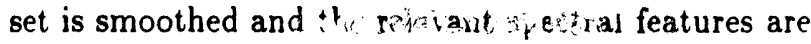
extracted. A feature unsisis of two components: the frequency of a resonati nese and a measure of the sharpness of the pcak. Fice figzures from all munitions in the training set ar's cluftered so that the variance of each cluster is mi mized. These clusters form. a template for a particia $e^{\prime}$ murition. When an unknown munitios is ats its spectral features are extracted and compares with a library of available templates. If the spectral feacures of the munition and a template in the library are closely matched, the munition is declared to belong to the class associated with that template. In the sequel, we describe the classification technique in detail. 


\section{Measurement System}

The acoustic spectra of a munition is measured by a system composed of a signal synthesizer/receiver, digitizer, a pair of piezoelectric transducers, and a control computer. The frequency synthesizer generates a stepped frequency sinusoid from $f_{\text {start }}$ to $f_{\text {otop }}$ where $f_{\text {start }}$ is typically set to $1 \mathrm{kHz}$ and $f_{\text {stop }}$ is typically set to $30 \mathrm{kHz}$. The frequency increment of the stepped sinusoid is adjustable, but nominally set to $f_{\text {inc }}=25 \mathrm{~Hz}$ and the dwell time on each tone is $1 / f_{\text {ine }}$ seconds. A piezoelectric transducer couples the energy from the signal synthesizer into the munition. The power applied to the transducer is approximately $1 \mathrm{~mW}$, which induces vibrations in the munitions with amplitudes on the order of 10 nanometers. A second piezoelectric transducer is used to receive the response of the munition. The output signal is quadrature demodulated with a homodyne receiver, and the in-phase and quadrature-phase baseband signals are digitized with 14 bits of resolution. The piezoelectric transducers are housed in a hand held mount that magnetically attaches itself to a munition. The entire system is small, portable, and is battery powered for up to five hours of continuous field use. Finally, the control computer has a custom-built user interface that controls operation of the entire system.

\section{Pattern Recognition Algorithms}

Several different algorithms are described here. The algorithms are used for two purposes: to construct templates from known munitions and to test unknown munitions. Templates are constructed by collecting spectral data from ten to twenty known munitions, extracting spectral features from the data and clustering the features to form a template for that class of munition. Unknown munitions are classified by measuring the unknown's acoustic spectrum, extracting its spectral features and comparing the features to a library of templates. The algorithms used for these tasks are described below.

\subsection{Feature Extraction}

A raw measurement is a sequence of $N$ complexvalued samples of spectral data $\tilde{X}_{r}(n)+j \tilde{X}_{i}(n)$, where $n=0, \ldots, N-1$ and $N=\left(f_{\text {stop }}-f_{\text {start }}\right) / f_{\text {inc }}$. An $M$-point unity-area Hamming window $w(n)$ is convolved with the raw data to produce a smoothed version $X(n)=\tilde{X}(n) * w(n)$. In order to increase the statistical reliability of the measurement, the length of the window is set so that $M \gg 1$. For a typical measurement, the value of $M$ is set to $M=32$. The magnitude of the smoothed data, $S(n)=\sqrt{X(n) X^{*}(n)}$, is computed and then normalized. Figure 1 illustrates a normalized magnitude plot of raw data, and Figure 2 illustrates a plot of processed data.

Feature extraction consists of locating peaks in the processed spectral data, determining the frequencies of the peaks, $f_{i}$, and their quality factors, $q_{i}$. The frequencies of the peaks are computed by firstorder differencing the processed spectral data $S^{\prime}(n)=$ $S(n)-S(n+1), 0 \leq n \leq N-2$, and then locating zero crossings in the difference sequence. The frequencies at which the difference sequence $S^{\prime}(n)$ is equal to zero correspond to peaks in $S(n)$. The current implementation finds the first 80 peaks, a number that has been found to be adequate. Figure 3 illustrates the locations of peaks extracted from a measurement superimposed over the processed spectrum.

The quality factor of the $i^{t^{t h}}$ peak, $q_{i}$, is determined as follows. We define the quality factor $Q$ of a peak to be $Q=H / B$ where $H$ is the height of the peak and $B$ is the width of the base of the peak. The value of $B$ of the $i^{\text {th }}$ peak is determined by starting at the frequency of the peak, $f_{i}$, and searching in the direction of increasing frequency for the frequency $f_{u}$ associated with first valley above the peak. The same search is performed in the direction of decreasing frequency for the frequency $f_{l}$ associated with the first valley below the peak. The width of the base is defined as $B=2 \times \min \left(\left|f_{i}-f_{u}\right|,\left|f_{i}-f_{i}\right|\right)$. The height $H$ is defined to be the difference between the magnitude of the peak and the magnitude of the closest valley, $H=S\left(f_{i}\right)-S\left(f_{v}\right)$ where $f_{v}$ is either $f_{u}$ or $f_{l}$, whichever corresponds to the frequency used in the width calculation.

Since a measurement typically contains many features, some of which are not significant, only features with large values of $Q$ are accepted for further processing. All other features are rejected as being insignifcant. The features are sorted in order of increasing $q_{i}$, and the first 50 features are selected. Thus, the feature extraction algorithm produces a set of features where the $i^{\text {th }}$ feature consists of a frequency and a quality factor, $\left(f_{i}, q_{i}\right)$. Figure 3 illustrates the output of the feature extraction algorithm for the data depicted in Figure 2.

\subsection{Clustering and Template Construc- tion}

The feature extraction algorithm generates a set of $N$ features $F=\left\{\left(f_{i}, q_{i}\right), i=1,2, \ldots N\right\}$ for each 
measurement. To build a template, measurements on multiple objects are required. Thus, a featuresct $F_{j}$, where $j=1,2, \ldots M$ is constructed from the $M$ measurements. A clustering algorithm is used to for...2 clusters of features so that templates for the object class can be constructed. The idea behind the clustering algorithm is to form clusters with the smallest possible variance. A brute force implementation of this approach is computationally complex if the featureset is large, but modifications of the basic idea allow more tractable solutions.

The computational complexity of the clustering algorithm is reduced by forming clusters from features within a narrow frequency range. Only features that are close in frequency are clustered. Of these clusters, only the ones with minimum variance are accepted. Specifically, the clustering algorithm begins by finding the maximum and minimum frequencies in the featureset $F_{j}, f_{\max }=\max _{f_{i}}\left\{F_{j}\right\}$ and $f_{\min }=\min _{f_{1}}\left\{F_{j}\right\}$. A frequency window that has a frequency span that is a fraction of the frequency range of the featureset is computed $\Delta W=W_{f}\left(f_{\max }-f_{\min }\right)$, where $W_{f}$ is a scaling factor typically set to 0.025 . The center frequency of the window is shifted by a small fraction of the window's width each iteration so that $W_{k}=f_{\min }+k W_{i} \Delta W+\Delta W / 2$ where $W_{i}$ is an increment factor set to $W_{i}=0.1$. The increment index $k$ is restricted to the range $0 \leq k \leq N_{w}$ where $N_{w}=\frac{1}{W_{1}}\left(\frac{1}{W_{\rho}}-1\right)-1$.

Once the window is in place, the featureset is scanned to find features that lie within the window. A family of sets $C_{k}$ is formed such that $C_{k}=\left\{\left\{\left(f_{i}, q_{i}\right)\right\}\right.$ : $\left.\left(f_{i}, q_{i}\right) \in F_{j}, W_{k}-\Delta W / 2 \leq f_{i} \leq W_{k}+\Delta W / 2\right\}$. If fewer than $80 \%$ of the measurements contribute features to $C_{k}$, the family of sets is abandoned, and $W_{k}$ advances to the next iteration. If the inclusion criterion has been met, the algorithm finds the set of features that produce the smallest frequency variance $\sigma_{k}^{2}=\min _{\sigma_{j}^{2}}\left\{C_{k}\right\}$. An exhaustive search is performed by forming all possible combinations of features, taking one feature from each measurement contained in $C_{k}$. Once the set of features with the smallest frequency variance has been found, the mean of the frequencies in the cluster is computed $f_{k}$, and features that have frequencies less than the mean frequency $f_{i}<f_{k}$ are prohibited from forming new clusters. This action prevents features that are on opposite fringes of a cluster from combining into a new cluster.

Four quantities are derived from each cluster: the mean of the frequencies in the cluster $f_{k}$, the standard deviation of the frequencies in the cluster $\Delta f_{k}$, the mean of the quality factors in the cluster $q_{k}$, and the standard deviation of the quality factors in the cluster $\Delta q_{k}$. These four quantities $\left(f_{k}, \Delta f_{k}, q_{k}, \Delta q_{k}\right)$ adequately characterize the cluster for classification purposes. It has been observed that clusters tend to be narrow in frequency relative to quality factor. Ideally, clusters would be narrow in both quantities. Additional research is required to develop better algorithms for finding a quality factor that narrows the clusters in this dimension, or identify other types of features that provide more compact clusters.

After all clusters have been found, several selection criteria are applied to determine the clusters used to construct a template. First, the widths of the clusters in frequency are sorted into ascending order, $\Delta f_{1}<\Delta f_{2}, \ldots, \Delta f_{N}$, where $N$ is the number of clusters constructed. The sequence of cluster widths are first-order differenced, and a running mean of the differences is computed. The reasoning behind this procedure is the observation that if the frequency widths of the clusters are listed in increasing order, a large jump in magnitude often occurs after the first few clusters. The location of this jump provides a demarcation between clusters associated with strong resonant peaks and clusters that the algorithm built out of smaller features. Clusters beyond the point where the next difference exceeds a threshold (four times the running mean) are excluded from the template. Clusters within the threshold are used as the template. Occasionally, no obvious jurnp occurs in the frequency width magnitude. In this case the first 16 clusters are used to construct the template. Figure 3 illustrates the clusters found by the clustering algorithm for five measurements on the same class of objects used in the previous figures.

\subsection{Munition Classification}

Templates are used to determine if an object, as represented by its measured features, belongs to the class of objects whose features were used to construct the template. Comparison between the features of an object and a template is performed by a template matching algorithm. The output of the algorithm is a number that represents the degree of match between the features extracted from a measurement and the template.

The template matching algorithm counts the number of frequency features from a measurement that are within frequency windows derived from cluster parameters in the template. Each frequency window is centered at the mean frequency of one entry in the template, and has a width equal to the width of the associated cluster. Once a feature has been counted 


\begin{tabular}{|l|cccccc|}
\hline & GH55 & HO55 & HE55 & S55V & VX55 \\
\hline GH55 & 0.777 & 0.222 & $\mathbf{0 . 6 6 6}$ & $\mathbf{0 . 2 7 7}$ & $\mathbf{0 . 1 6 6}$ \\
HO55 & 0.055 & $\mathbf{0 . 6 6 6}$ & $\mathbf{0 . 3 8 8}$ & $\mathbf{0 . 4 4 4}$ & 0.222 \\
HE55 & $\mathbf{0 . 1 6 6}$ & $\mathbf{0 . 2 2 2}$ & $\mathbf{0 . 5 5 5}$ & $\mathbf{0 . 2 2 2}$ & $\mathbf{0 . 2 2 2}$ \\
S55V & $\mathbf{0 . 1 1 1}$ & $\mathbf{0 . 4 4 4}$ & $\mathbf{0 . 1 6 6}$ & $\mathbf{0 . 4 4 4}$ & $\mathbf{0 . 0 5 5}$ \\
VX55 & $\mathbf{0 . 2 2 2}$ & $\mathbf{0 . 2 2 2}$ & $\mathbf{0 . 3 8 8}$ & $\mathbf{0 . 2 2 2}$ & $\mathbf{0 . 6 6 6}$ \\
\hline
\end{tabular}

Table 1: Hit ratios for a single measurement of a munition type (rows) for munition templates (columns).

as a "hit," it is prohibited from contributing to hits on other wirdows. After all features have been scanned for hits, a hit ratio, i.e. the ratio of the number of features in the measurement that match features in the template to the number of features in the template, is computed. The larger the hit ratio the better the match between the features of a measurement and the template. In order to account for possible frequency shifts of a measurement's features, which can occur if the fill level of the munition differs from those used to construct the template, the frequency windows are shifted by a slight amount (three times the bin width of the measurement) and the hit ratio is again computed. The hit ratio for each shift is computed, and the largest hit ratio of all shifts is reported.

\section{Preliminary Results}

The algorithms presented in this paper were developed and tested with ARS data collected at the Tooele Army Depot. Although the lack of data prevented a statistical analysis of the algorithms' performance, two examples are given that illustrate the type of result$s$ we obtained with the algorithms. The designators, shell sizes and compositions of the shells discussed in the examples are: GH55, $155 \mathrm{~mm} \mathrm{~GB}$ nerve agent (high purity); HO55, $155 \mathrm{~mm}$ Mustard gas; HE55, $155 \mathrm{~mm}$ high explosive; VX55, $155 \mathrm{~mm}$ VX nerve agent; S55V, $155 \mathrm{~mm}$ VX physical surrogate (designed to match the mechanical properties of VX55).

In the first example, a template for each munition class was constructed using seven randomly selected measurements from each class. Another randomly selected measurement from each class was classified against the templates. The hit ratio for each template is listed in Table 1.

The example illustrates how the features from a particular munition match a template from the same class of munition and also match templates created from other munitions. The only ambiguous result occurs when the $555 \mathrm{~V}$ measurement matches the HO55 and $\mathrm{S} 55 \mathrm{~V}$ templates equally well. The classification error is probably caused by a bad measurement, which in turn might be due to poor transducer placement or poor transducer coupling.

As another example, five measurements from each munition class were compared to the five templates. The GH55, HE55, and S55V shells were correctly identified on all five trials. The HO55 and VX55 shells were correctly identified on four of the five trials and misidentified once each. While these results do not represent an exhaustive test of the algorithms, they are indicative of the results we have obtained. In the vast majority of trials, a munition matched the template of its class either best or second best.

\section{Summary}

The ARS classification technique described in this paper has been field tested on a wide variety of munitions. The technique is based on the acoustic resonance structure of the munition and has shown good discrimination ability. A field instrument constructed to implement the technique will be used by the On Site Inspection Agency to verify compliance with the Bilateral Chemical Weapons Agreement between the United States and Russia.

\section{References}

[1] D. N. Sinha, "Acoustic Resonance Spectroscopy," IEEE Potentials, April 1992, vol. 11, No. 2, pg. 10. 


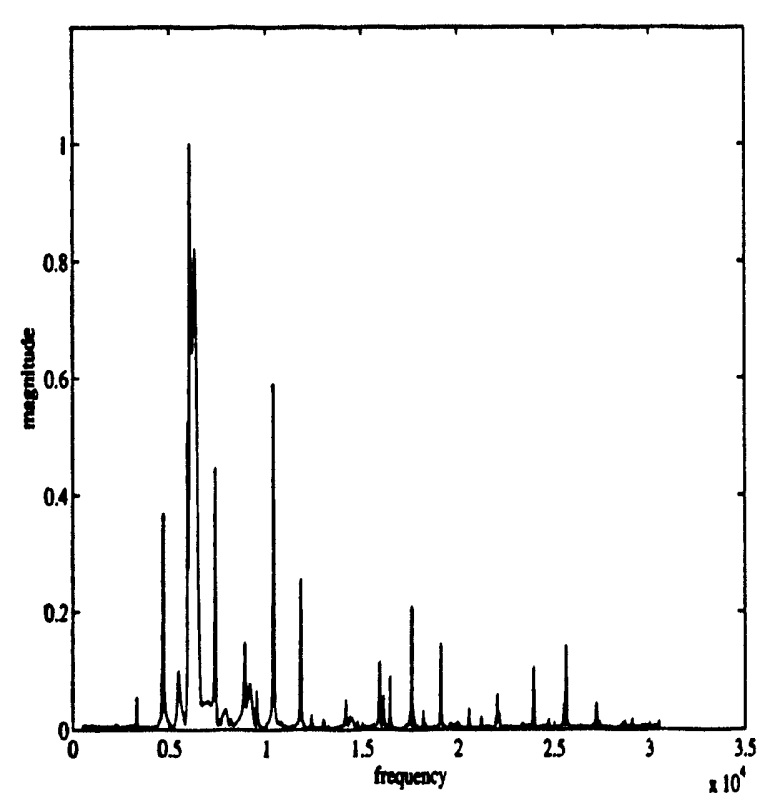

Figure 1: Normalized magnitude plot of raw acoustic spectral data.

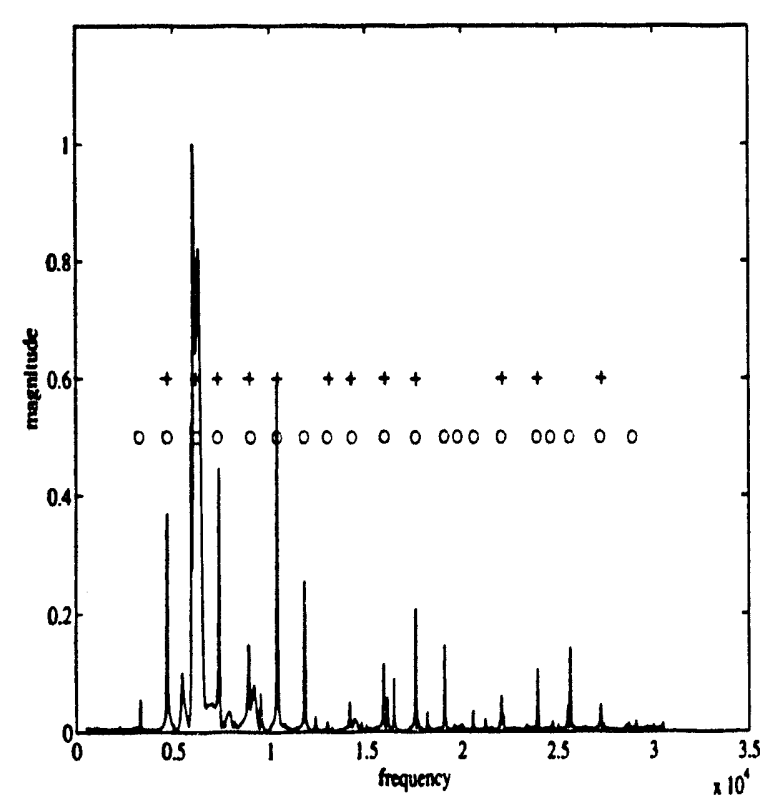

Figure 3: Normalized magnitude plot of raw spectral data with extracted features denoted by ' $O$ ', and features selected by the clustering algorithm denoted by 't'.

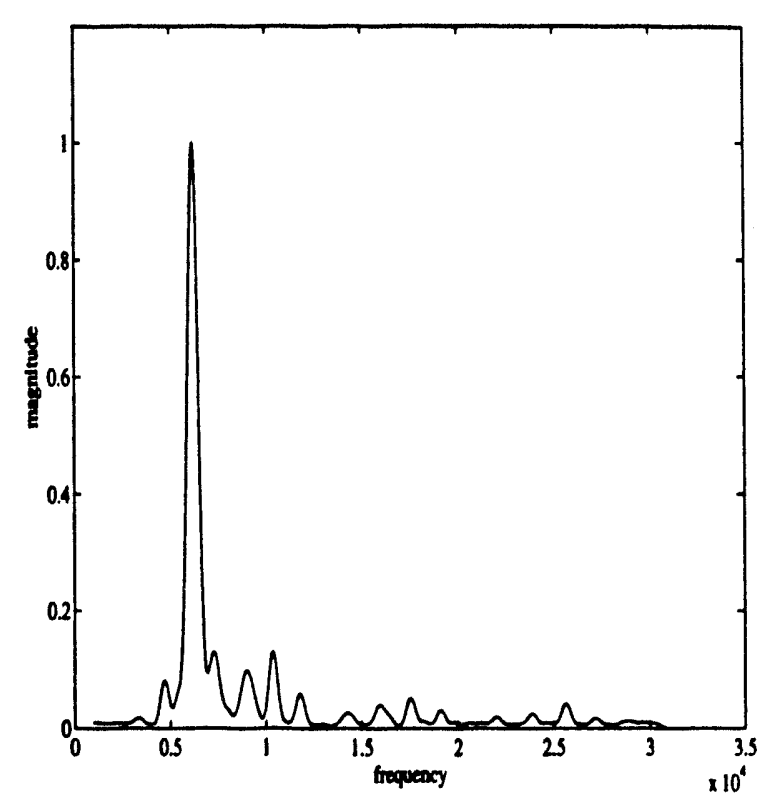

Figure 2: Raw acoustic data smoothed with a 32 point, unity-area Hamming window.

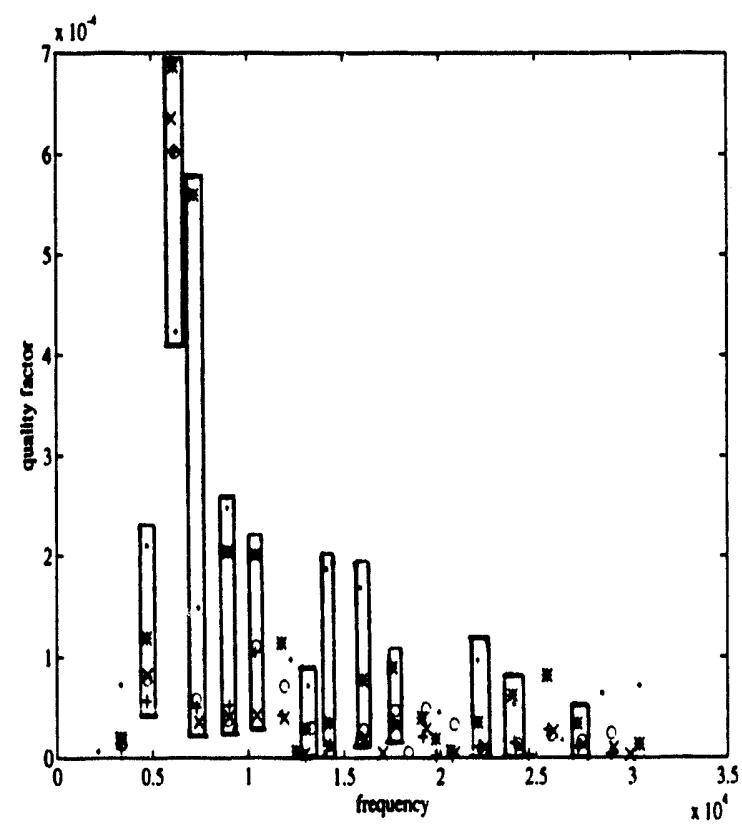

Figure 4: Plot of the featureset of five measurements. Clusters found by the clustering algorithm are circled.

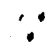




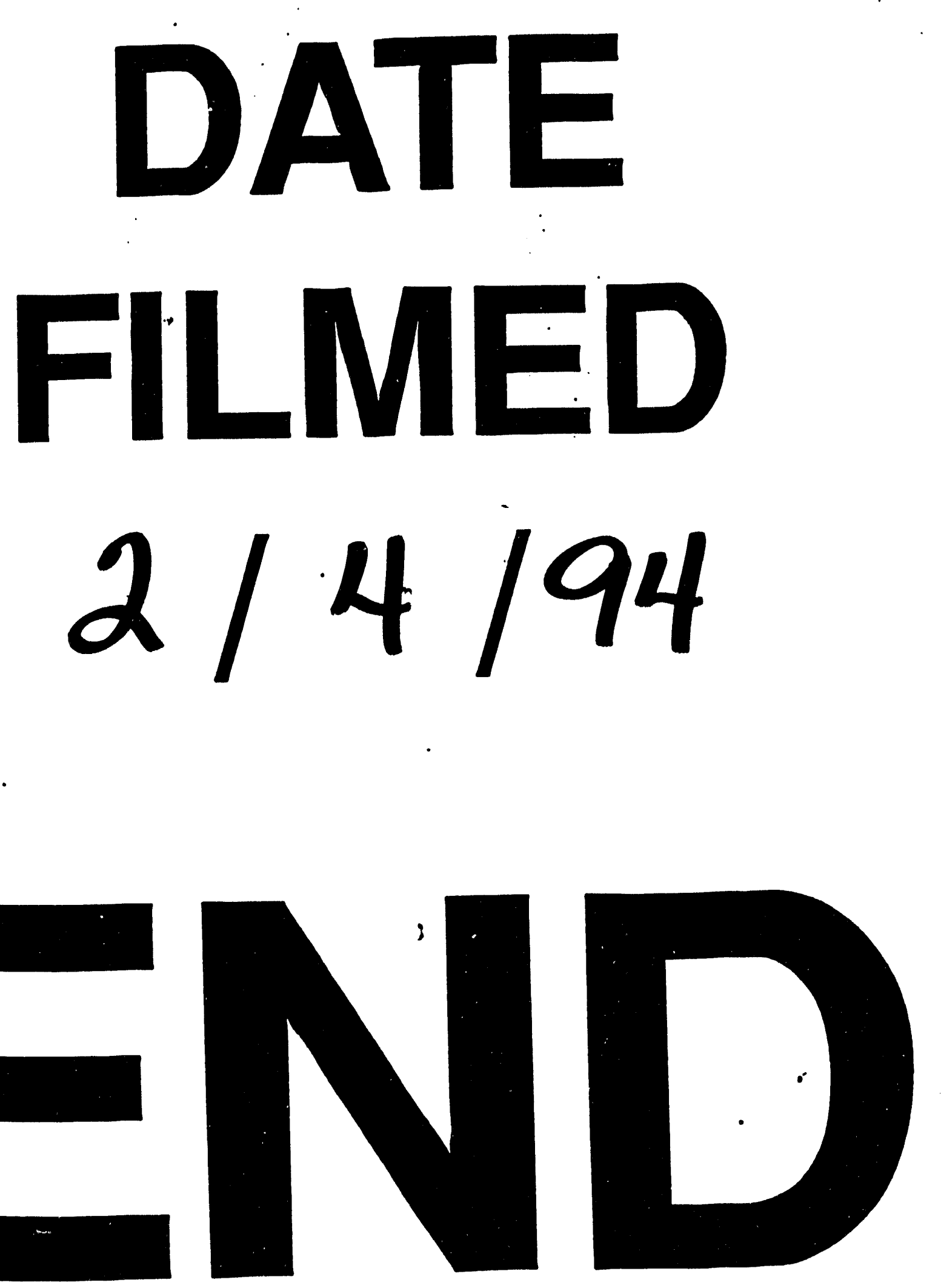


